\title{
The role of radiative losses in the late evolution of pulse-heated coronal loops/strands
}

\author{
F. Reale ${ }^{1,2}$ and E. Landi ${ }^{3}$ \\ 1 Dipartimento di Fisica, Università di Palermo, Piazza del Parlamento 1, 90134 Palermo, Italy \\ e-mail: reale@astropa.unipa.it \\ 2 INAF - Osservatorio Astronomico di Palermo, Piazza del Parlamento 1, 90134 Palermo, Italy \\ 3 Department of Atmospheric, Oceanic and Space Sciences, University of Michigan, Ann Arbor, MI 48109, USA
}

Received 23 March 2012 / Accepted 19 May 2012

\begin{abstract}
Context. Radiative losses from optically thin plasma are an important ingredient in modeling confined plasma in the solar corona. Spectral models are continuously updated to include the emission from more spectral lines, with significant effects on radiative losses, especially around $1 \mathrm{MK}$.

Aims. We investigate the effect of changes to the radiative-loss temperature dependence caused by upgrading spectral codes on the predictions obtained when modeling confined plasma in the solar corona.

Methods. We revisit the hydrodynamic simulation of a pulse-heated loop strand by comparing results obtained using an old and a recent radiative-loss function.

Results. We find that significant changes occur in the plasma evolution during the late phases of plasma cooling: when the more recent radiative-loss curve is used, the plasma cooling rate indeed increases significantly when temperatures reach 1-2 MK. This more rapid cooling occurs when the plasma density is higher than a threshold value, which in impulsive heating models leads to the loop plasma becoming overdense. This rapid cooling has the effect of steepening the slope in the emission measure distribution of coronal plasmas with temperature, at temperatures lower than $\sim 2 \mathrm{MK}$, and of reducing the visibility of warm (1 MK) loops.

Conclusions. The effects of changes to the radiative-loss curves can be important when modeling the late phases of the evolution of pulse-heated coronal loops, and, in general, of thermally unstable optically thin plasmas.
\end{abstract}

Key words. Sun: X-rays, gamma rays - Sun: corona - Sun: UV radiation - Sun: activity - radiation mechanisms: thermal hydrodynamics

\section{Introduction}

Coronal loops are the building blocks of the solar corona. They consist of pipe-like magnetic field structures arching in the corona and connecting photospheric magnetic regions of opposite polarity. Loops are filled with optically thin, hot, and relatively dense plasma, with temperatures ranging from $0.8 \mathrm{MK}$ to a few million degrees, depending on the regions where they are located. There are two main classes of mechanisms that have been proposed to explain the heating of the loop plasma to coronal temperatures: steady (or "high frequency", Warren et al. 2011) heating mechanisms, and impulsive (or "low frequency") heating mechanisms. Loop models that include steady heating predict a steady-state plasma, while in the pulse-heated scenario the plasma evolves dynamically and spends most of the time in a cooling state (Cargill 1994). In particular, a fast energy pulse might heat the plasma to more than $10 \mathrm{MK}$ for a few seconds (e.g., Cargill 1994; Cargill \& Klimchuk 2004; Reale \& Orlando 2008; Guarrasi et al. 2010); the plasma is then free to cool, losing energy by both conduction to the chromosphere and radiation. The balance between these two loss mechanisms is essentially determined by the local density: during and immediately after the heat pulse, the loop plasma density is still low and conduction dominates, then denser plasma from the chromosphere fills the loop and radiation becomes the most efficient loss mechanism (Antiochos 1980; Cargill \& Klimchuk 2004; Reale $2007,2010)$. Observational evidence seems to indicate that loop plasma might be heated impulsively (as reviewed in Klimchuk 2006), and recent studies using Hinode and Solar Dynamics Observatory data seem to confirm this idea (Guarrasi et al. 2010; Reale et al. 2011; Terzo et al. 2011; Viall \& Klimchuk 2011).

Regardless of the heating scenario, radiative losses are an important mechanism of plasma cooling in coronal loops and need to be included to high accuracy in loop models (e.g., McClymont \& Canfield 1983; Antiochos \& Sturrock 1982; Bradshaw \& Mason 2003; Müller et al. 2003; Bradshaw \& Cargill 2005). They become particularly important if loops are described as bundles of thin strands (Cargill 1993; Klimchuk 2006), each ignited by a single short and intense heat pulse (Parker 1988), as the plasma cooling times are far longer than the expected duration of the heat pulse so that each strand spends most of its time cooling by radiation. Radiative losses are calculated by summing the emission of the plasma over the entire wavelength spectrum. The radiative emission of the optically thin plasma is dominated by the bremsstrahlung and free-bound recombination continua, and by line emission from all ions of all elements present in the coronal plasma. The total radiative losses are approximately proportional to the square of the electron density of the plasma (Tucker \& Gould 1966; Landini \& Monsignori Fossi 1970; Tucker \& Koren 1971); Landi \& Landini (1999) indeed 
showed that departures from this proportionality are smaller than $25 \%$. The dependence of these losses on the electron temperature, in contrast, is much more complex and, in typical coronal conditions, can be parameterized with a function obtained by fitting a known curve to the total radiative losses of isothermal spectra calculated using a grid of temperature values (Rosner et al. 1978; Landi \& Landini 1999).

The isothermal spectra used to determine the total radiativeloss curve are obtained using a spectral code, which collects all the relevant atomic parameters and transition rates necessary to calculate the line and continuum emission of an optically thin plasma. The most popular codes available in the literature are CHIANTI (Dere et al. 1997; Landi et al. 2012), AtomDB (Foster et al. 2012), and SPEX (Kaastra et al. 1996); as new and improved calculations of atomic data and transition rates become available in the literature, these codes are constantly updated to extend their calculations to lines that had previously been neglected, or improve the results for those previously considered. As a consequence, the predicted radiative losses can change, and the differences from those calculated with earlier versions of the codes can sometimes be large.

The consequences of upgrades to spectral codes and radiative losses can be important for both data analysis and diagnostics (Testa et al. 2012) and for plasma modeling (Soler et al. 2012). For example, time-dependent hydrodynamic plasma models have been extensively used to describe loop plasma evolution. All models include the radiative losses in the energy balance, sometimes with a temperature dependence described with a piecewise power-law function as in Rosner et al. (1978), which is based on the Raymond \& Smith (1977) spectral model. More recent calculations of the radiative losses have a much higher loss rate at around $1 \mathrm{MK}$, owing to the higher metal abundances and the inclusion of a large amount of spectral lines from Fe vIII-Xv as well as other elements formed at similar temperatures. The differences at higher temperatures are more limited. Thus, for a plasma impulsively heated to about $10 \mathrm{MK}$, we do not expect large differences for most of the evolution. However, when the plasma cools and approaches $1 \mathrm{MK}$, the much higher loss rate will cause the loop to cool more efficiently.

In this paper, we show in detail that in a pulse-heated loop model, the enhanced radiative losses more easily and earlier lead to catastrophic cooling, which qualitatively changes the plasma evolution and may have important implications for both the observed emission distribution in active and quiet regions and the loop heating mechanisms. Our approach is to revisit a welltested multi-strand pulse-heated loop model (Reale \& Orlando 2008; Guarrasi et al. 2010) using both a standard and an updated radiative-loss function and discuss the differences in our results.

Section 2 summarizes the loop model focusing on the radiative-loss function, Sect. 3 illustrates our results for different functions, Sect. 4 describes the implications for diagnostics, and Sect. 5 discusses our results and their implications.

\section{The model}

We consider a hydrodynamic simulation identical to the one in Guarrasi et al. (2010), which models a pulse-heated loop strand with the Palermo-Harvard loop code (Peres et al. 1982; Betta et al. 1997). The loop strand is semicircular and symmetric with respect to the apex, and its symmetry axis is perpendicular to the solar surface. The loop half-length is $L=3 \times 10^{9} \mathrm{~cm}$ and includes a chromospheric layer at the footpoints that is linked to the corona through a steep transition region.
The plasma confined in each strand transports energy and moves only along the magnetic field lines, and its evolution can be described with a one-dimensional time-dependent hydrodynamic model (e.g., Nagai 1980; Peres et al. 1982; Doschek et al. 1982; Nagai \& Emslie 1984; McClymont \& Canfield 1983; MacNeice 1986; Gan et al. 1991; Hansteen 1993; Betta et al. 1997; Antiochos et al. 1999; Müller et al. 2003; Bradshaw \& Mason 2003; Bradshaw \& Cargill 2006), through the equations (Peres et al. 1982; Betta et al. 1997)

$$
\begin{aligned}
& \frac{\mathrm{d} n}{\mathrm{~d} t}=-n \frac{\partial v}{\partial s} \\
& n m_{\mathrm{H}} \frac{\mathrm{d} v}{\mathrm{~d} t}=-\frac{\partial p}{\partial s}+n m_{\mathrm{H}} g+\frac{\partial}{\partial s}\left(\mu \frac{\partial v}{\partial s}\right) \\
& \frac{\mathrm{d} \varepsilon}{\mathrm{d} t}+w \frac{\partial v}{\partial s}=Q-n^{2} \beta P(T)+\mu\left(\frac{\partial v}{\partial s}\right)^{2}+\frac{\partial}{\partial s}\left(\kappa T^{5 / 2} \frac{\partial T}{\partial s}\right) \\
& p=(1+\beta) n k_{\mathrm{B}} T \\
& \varepsilon=\frac{3}{2} p+n \beta \chi \\
& w=\frac{5}{2} p+n \beta \chi
\end{aligned}
$$

where $n$ is the hydrogen number density, $t$ is time, $s$ is the field line coordinate, $v$ is the plasma velocity, $m_{\mathrm{H}}$ is the mass of hydrogen atom, $p$ is the pressure, $g$ is the component of gravity along the field line, $\mu$ is the effective coefficient of compressional viscosity (including numerical viscosity), $\beta=n_{\mathrm{e}} / n$ is the ionization fraction where $n_{\mathrm{e}}$ is the electron density, $T$ is the temperature, $\kappa$ is the thermal conductivity $\left(\simeq 9 \times 10^{-7} \mathrm{erg} \mathrm{cm}^{-1} \mathrm{~s}^{-1} \mathrm{~K}^{-7 / 2}\right), k_{\mathrm{B}}$ is the Boltzmann constant, $\chi$ is the hydrogen ionization potential, $P(T)$ is the radiative-loss function per unit emission measure (discussed later), and $Q(s, t)$ is the power input per unit volume

$Q(s, t)=H_{0}+H_{1} f(t)$,

where $H_{0}$ is a low-regime $\left(H_{0}=3 \times 10^{-5} \mathrm{erg} \mathrm{cm}^{-3} \mathrm{~s}^{-1}\right)$ steady heating term that balances radiative and conductive losses for the static initial atmosphere, and $H_{1}$ is the amplitude of the heat pulse, which is assumed to be uniform along the loop. The time dependence of the heat pulse is a top-hat function, with $f(t)=1$ for $0<t<60 \mathrm{~s}$ and $f(t)=0$ at any other time. The amplitude of the pulse is $H_{1}=0.38 \mathrm{erg} \mathrm{cm}^{-3} \mathrm{~s}^{-1}$. We checked that the presence of the steady heating term $\left(H_{0}\right)$ is irrelevant to the entire strand evolution. For further investigation, we also show simulations for different intensities of the heat pulse.

The initial condition is that of a very low-pressure loop at-

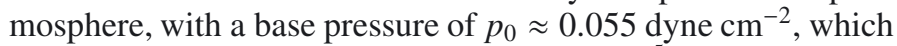
results in an apex temperature of $T_{0} \approx 8.0 \times 10^{5} \mathrm{~K}$. The chromosphere is assumed to be model $\mathrm{F}$ in Vernazza et al. (1981), and its energy balance is strictly maintained at all times.

The Palermo-Harvard loop code (Peres et al. 1982; Betta et al. 1997) has been extensively used to model both flaring (Peres et al. 1987; Betta et al. 2001) and quiescent loops (Reale et al. 2000; Guarrasi et al. 2010). The code has an adaptive mesh refinement (Betta et al. 1997), to adequately achieve high resolution in the steep gradients along the strand and during the evolution.

We replicate the simulation of Guarrasi et al. (2010) for two different radiative-loss functions $P(T)$, shown in Fig. 1: one for Rosner et al. (1978, hereafter RTV) computed according to Raymond \& Smith (1977), the other computed according to version 7 of the CHIANTI code (Landi et al. 2012), assuming a density of $10^{9} \mathrm{~cm}^{-3}$ and ionization equilibrium according to 
F. Reale and E. Landi: Radiative losses in pulse-heated coronal loops

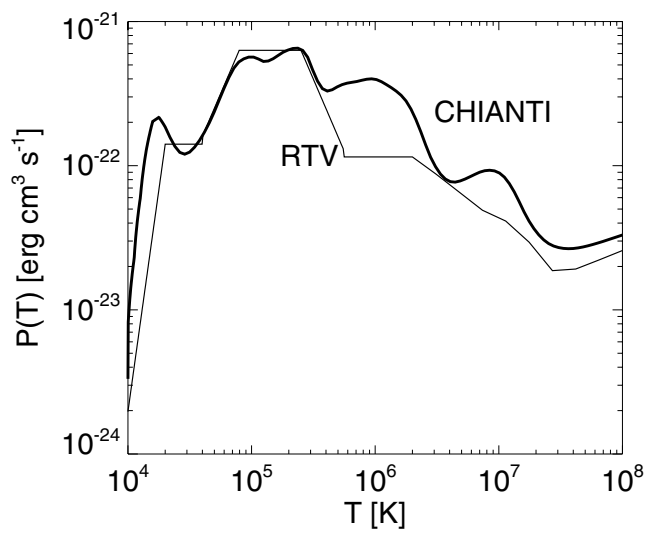

Fig. 1. Radiative-loss function $P(T)$ (emissivity per unit emission measure) as a function of temperature according to Rosner et al. (1978) (thin solid line) and to version 7 of the CHIANTI spectral code (Landi et al. 2012) (thick solid line).

Dere (2009). We checked that radiative-loss functions adopted in other works, e.g. Klimchuk et al. (2008), fall in the range between these two curves and therefore we expect intermediate results when using them.

The differences between the two curves have three sources. First, the atomic models in CHIANTI are much more extensive in terms of line coverage and more sophisticated than those available to Raymond \& Smith (1977), and such larger atomic models provide vast amounts of additional spectral lines. The main differences fall around $0.5-3 \mathrm{MK}$, because of the increase in the size of the Fe VIII to Fe XIV models, and around $10 \mathrm{MK}$, because of the larger models for Fe XVIII-XXIII. Increases in the size of the models for ions of other elements have a more limited effect. Overall, Landi \& Landini (1999) found that increases in CHIANTI version 2 atomic models and improvements to the atomic data caused radiative losses to change by as much as $50 \%$. As even bigger models are used in CHIANTI version 7 , we expect differences to be larger.

The second source of variation lies in the ion fractions used in the two calculations. Ionization and recombination rates used to calculate the charge state composition at equilibrium are normally taken from theoretical calculations, which have been dramatically improved over the 30-40 years separating the Raymond \& Smith (1977) and the latest version of the CHIANTI code. The differences due to ion fraction improvements are expected to affect all elements and ions. Landi \& Landini (1999), for example, estimated that different ion abundance datasets caused the radiative losses to change by up to $40 \%$.

The third source of variation are element abundances. These are expected to provide large effects: Landi \& Landini (1999) reported differences of factors of up to 2.5 when different abundance datasets are used. In the present comparison, the abundances used by Raymond \& Smith (1977) are the cosmic values reported by Allen (1973), while the CHIANTI code radiative losses were calculated using the coronal abundances determined by Feldman et al. (1992). The main difference between the two abundance datasets is that the cosmic abundances of elements for which first ionization potential (FIP) is lower than $10 \mathrm{eV}$ have been increased in the coronal abundance dataset by a factor $\approx 3.5$ to account for the element fractionation in the solar corona known as the FIP effect (Feldman 1992); additional, much smaller differences are found between the photospheric element abundances of ions with FIP $>10 \mathrm{eV}$ and the cosmic values. The factor of 3.5 enhancement involves most of the
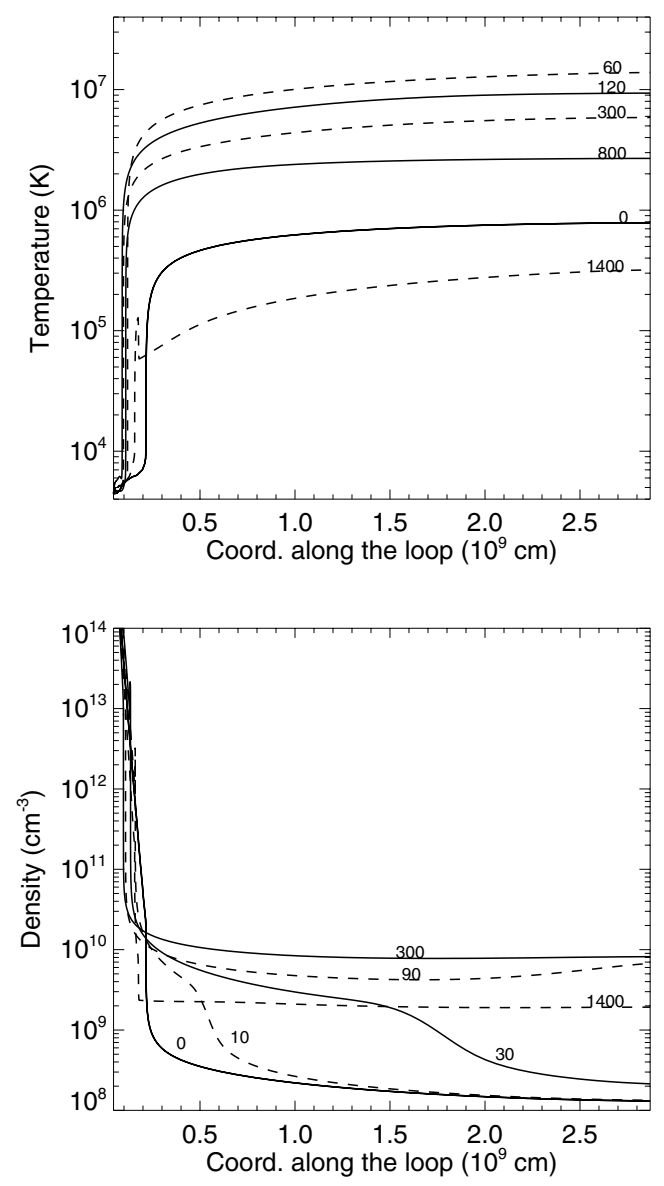

Fig. 2. Model temperature (top) and density (bottom) along half of the pulse-heated loop strand. The loop apex is at the right end of the $X$-axis. The plasma temperature and densities are displayed at several different times (in s) as reported in the two panels, from $0 \mathrm{~s}$ (heat pulse start) to $1400 \mathrm{~s}$.

major elements emitting in the corona - $\mathrm{Mg}, \mathrm{Si}$, and $\mathrm{Fe}$ - so that large effects are expected at temperatures in the 1-15 MK, where $\mathrm{Fe}$ emission dominates the spectrum.

\section{The results}

We calculated the evolution of the loop-confined plasma for $\sim 2000 \mathrm{~s}$ after the start of the impulsive heating. This evolution is known from many previous studies (e.g., Peres et al. 1993; Warren et al. 2002, 2003; Patsourakos \& Klimchuk 2005; Reale \& Orlando 2008; Guarrasi et al. 2010). Figure 2 shows samples of the temperature and density profiles along half of the strand at several different times $(0 \mathrm{~s}, 10 \mathrm{~s}, 30 \mathrm{~s}, 60 \mathrm{~s}, 90 \mathrm{~s}$, $120 \mathrm{~s}, 300 \mathrm{~s}, 800 \mathrm{~s}$, and $1400 \mathrm{~s}$ ) that encompass the entire strand evolution. Figure 2 displays the simulation computed with the CHIANTI radiative losses, but the evolution obtained using the other loss curve is overall very similar. In accordance with the strong heating pulse, the plasma rapidly heats to above $10 \mathrm{MK}(60 \mathrm{~s})$ along most of the loop. It then gradually and uniformly cools, reaching $\sim 0.1 \mathrm{MK}$, i.e. below the initial temperature, in about half an hour. The density follows a slightly different evolution on a different timescale. It increases initially with an evaporation front coming up from the chromosphere $(t=10 \mathrm{~s}$, $30 \mathrm{~s})$. After the front has reached the loop apex, the density increases more uniformly and reaches its maximum after about 5 min, i.e. much later than the end of the heat pulse. The density then also begins to decrease, owing to the draining driven by the 

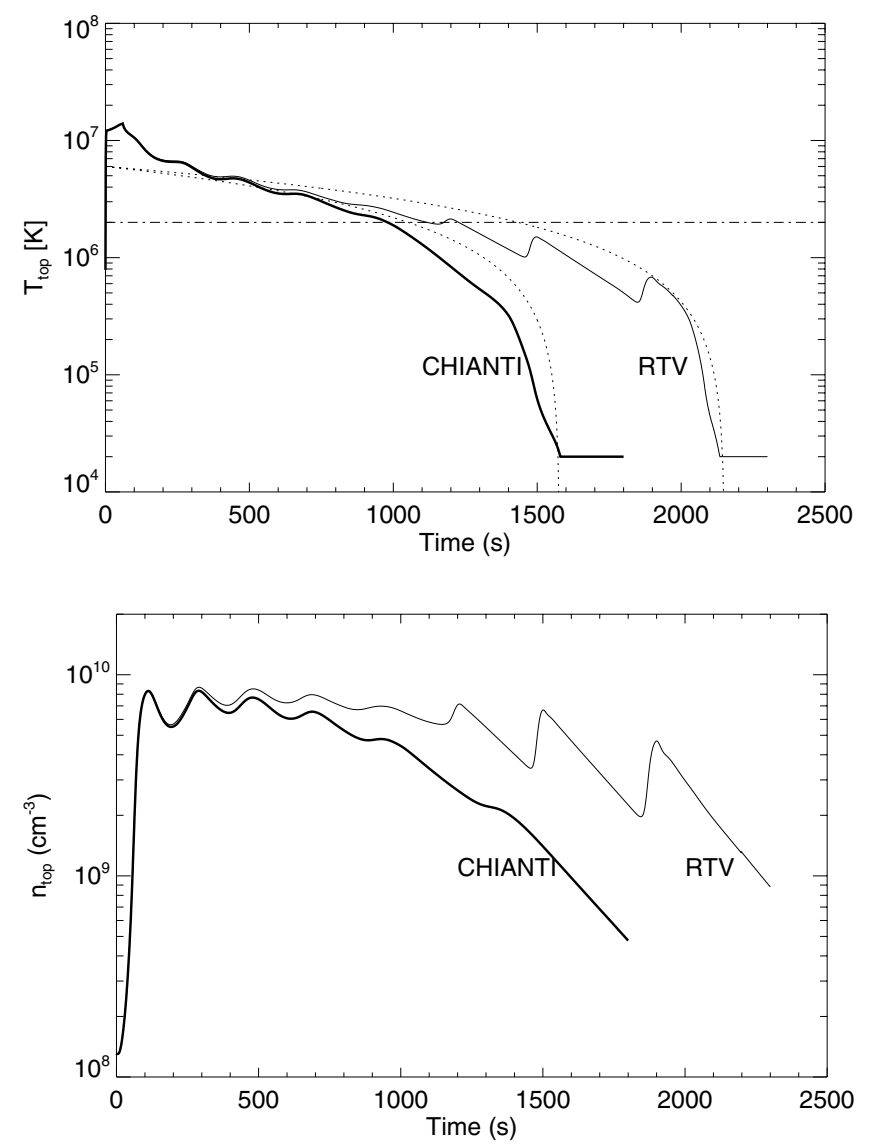

Fig. 3. Evolution of the temperature (top) and density (bottom) at the loop apex from hydrodynamic simulations using the two different radiative-loss functions: Rosner et al. (1978) (thin solid line) and version 7 of CHIANTI (thick solid line). Results obtained with analytical approximations (Cargill 1994) are also shown (dotted lines, see text for details). The level of $2 \mathrm{MK}$ is marked (dash-dotted horizontal line).

cooling (Bradshaw \& Cargill 2010). After about half an hour, the density is still much higher than it was before the heat pulse.

The effect of the different radiative-loss functions is more significant at relatively late times. This difference is illustrated in Fig. 3, which shows the evolution of the temperature and density at the loop apex obtained using the two different loss functions. Both temperature and density at the loop apex are the same during the first ten minutes of evolution, when the temperature is higher than $\simeq 2 \mathrm{MK}$. After $10 \mathrm{~min}$, the evolutionary tracks diverge: the plasma temperature and density calculated with the CHIANTI radiative losses decrease faster.

The temperature evolution ends with catastrophic cooling (Parker 1953; Field 1965) in both cases, with the temperature dropping abruptly by almost one order of magnitude to the minimum of $\sim 2 \times 10^{4} \mathrm{~K}$ in little more than $3 \mathrm{~min}$. Analytical approximations of the rate of decrease in the plasma temperature have been derived in the past (Antiochos 1980; Cargill 1994, 1995) and can be compared to those obtained numerically. Figure 3 shows two solutions obtained using the analytical expression (Cargill 1994)

$T_{\text {top }}(t)=T_{0}\left[1-\frac{3}{2}\left(\frac{1}{2}-\alpha\right) \frac{t}{\tau_{r}}\right]^{1 /(1 / 2-\alpha)}$,

where

$\tau_{r}=\frac{3 k_{\mathrm{B}} T_{0}}{n_{0} P(T)_{0}}$.
For both cases, we assumed $n_{0}=7 \times 10^{9} \mathrm{~cm}^{-3}$ as a density value, $T_{0}=6 \mathrm{MK}$ as a starting temperature of the radiative phase, from inspection of the simulations (see Fig. 3), and $\alpha=-0.5$ as an effective power-law index of the radiative-loss function approximated by $P(T)=\chi T^{\alpha}$ in the temperature range of interest, i.e. $5.5<\log T<7$ (Cargill 1994). We checked that the chosen values of $n_{0}$ and $T_{0}$ agree with those obtained by equating the thermal conductive and radiative cooling timescales (Cargill $\&$ Klimchuk 2004). The two solutions that closely match those of the simulations are obtained using two different values of the effective radiative losses, i.e. $P(T)_{0}=1.5 \times 10^{-22}$ and $1.1 \times 10^{-22} \mathrm{erg} \mathrm{cm}^{3} \mathrm{~s}^{-1}$ (see Fig. 1). Substituting in Eq. (9), we obtain $\tau_{r} \approx 2400 \mathrm{~s}$ and $3200 \mathrm{~s}$, for the faster (CHIANTI) and slower (RTV) cooling, respectively. The decay after the initial impulsive evolution, i.e. as soon as the temperature settles to about $6 \mathrm{MK}$, is generally well-described by Eq. (8), except for some details. In particular, the catastrophic cooling that occurs at $\sim 0.3 \mathrm{MK}$ is well-reproduced. The numerical solution with CHIANTI predicts a faster decay below $\sim 2 \mathrm{MK}$, leading to catastrophic cooling at $t \sim 1400 \mathrm{~s}$, i.e. $\sim 600 \mathrm{~s}$ earlier than with RTV losses. This corresponds approximately to the two important changes in the slope of the CHIANTI radiative-loss function (Fig. 1) at $T \sim 3 \mathrm{MK}$ and $0.5 \mathrm{MK}$. Therefore, the simulations are in good agreement with the analytical descriptions, in which we change only the "effectiveness" of the radiative losses in a certain temperature range, i.e. the average values of $P(T)$.

The density decreases more gradually than the temperature (Bradshaw \& Cargill 2010). In the RTV solution, we note the saw-toothed behavior due to plasma bouncing back and forth along the closed loop during draining. For our purposes, it is more important to consider the average evolution. The density obtained with CHIANTI losses decreases, on average, considerably faster than with RTV losses. The basic reason is that the more effective cooling makes the pressure decrease faster in absolute value, and the pressure gradient, i.e. the force that sustains the plasma against draining by gravity, decreases faster as well, causing a faster draining. At this point, the process is highly non-linear, i.e. catastrophic, and cannot be stopped, even if the radiative cooling rate decreases with the density. Bradshaw \& Cargill (2010) show the effect in terms of enthalpy, i.e. a peak of radiative losses is followed by a sharp increase of enthalpy losses.

Overall, we might say that the density stays almost steady for more than $1500 \mathrm{~s}$ with RTV losses, while its reduction becomes significant after only about $1000 \mathrm{~s}$ with CHIANTI losses. This qualitative difference has important implications for the emission measure distributions.

A very important qualitative difference of using CHIANTI instead of RTV radiative losses is the switching from regimes where there is a transition to catastrophic cooling, to regimes where the rate of cooling remains roughly constant, i.e. both radiation and enthalpy losses increase smoothly with time, as shown in Bradshaw \& Cargill (2010). This corresponds to the change in the slope of the cooling occurring at about $2 \mathrm{MK}$. We have investigated in more detail the conditions required to trigger this change in the behavior. Figure 4 shows the evolution of the temperature and density at the loop apex obtained with the CHIANTI radiative losses and different values of the heat pulse intensity $H_{1}$. Higher $H_{1}$ values lead to both higher apex maximum temperature and higher density values. Figure 4 shows that the change of slope has a strong dependence on the density, regardless of the heat pulse intensity. The slope indeed does not change if the loop plasma density remains approximately below the equilibrium density of a loop at a maximum temperature 
F. Reale and E. Landi: Radiative losses in pulse-heated coronal loops
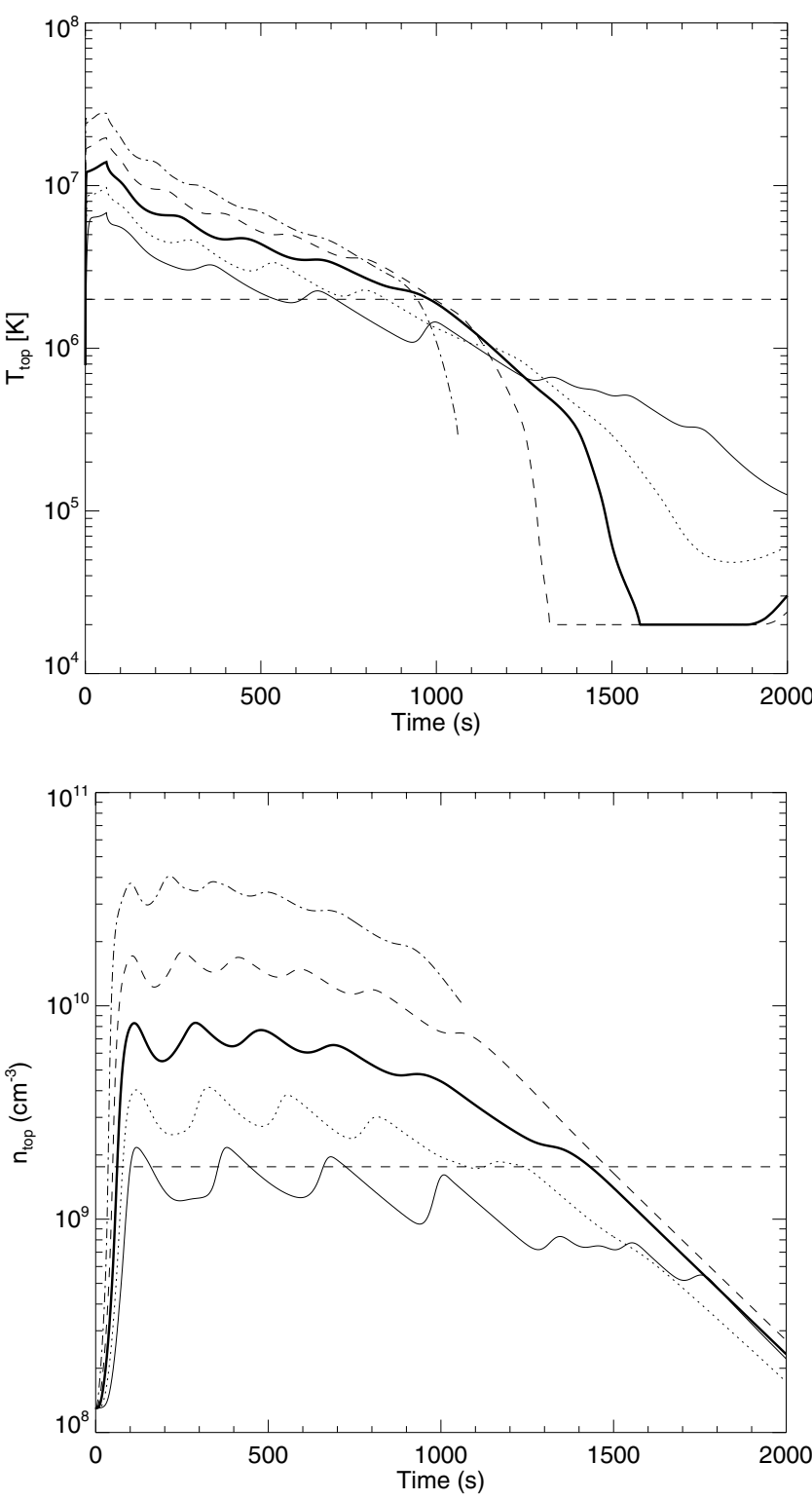

Fig. 4. Evolution of the temperature (top) and density (bottom) at the loop apex obtained with the CHIANTI radiative losses and different values of the heat pulse intensity. We indicate the density equilibrium value (dashed horizontal line, bottom panel) for a loop at $\sim 2 \mathrm{MK}$ (dashed horizontal line, top panel).

of about 2 MK. According to Rosner et al. (1978), this density threshold can be obtained as

$n_{9} \approx \frac{5}{L_{9}}$

where $n_{9}$ is the density in units of $10^{9} \mathrm{~cm}^{-3}$ and $L_{9}$ is the loop half-length in units of $10^{9} \mathrm{~cm}$. If the density is lower than this threshold value, then the loop does not enter a catastrophic cooling phase.

\section{Diagnostic implications of different radiative-loss curves}

The change in the cooling rate has two important consequences. First, an important diagnostic result that can be obtained from

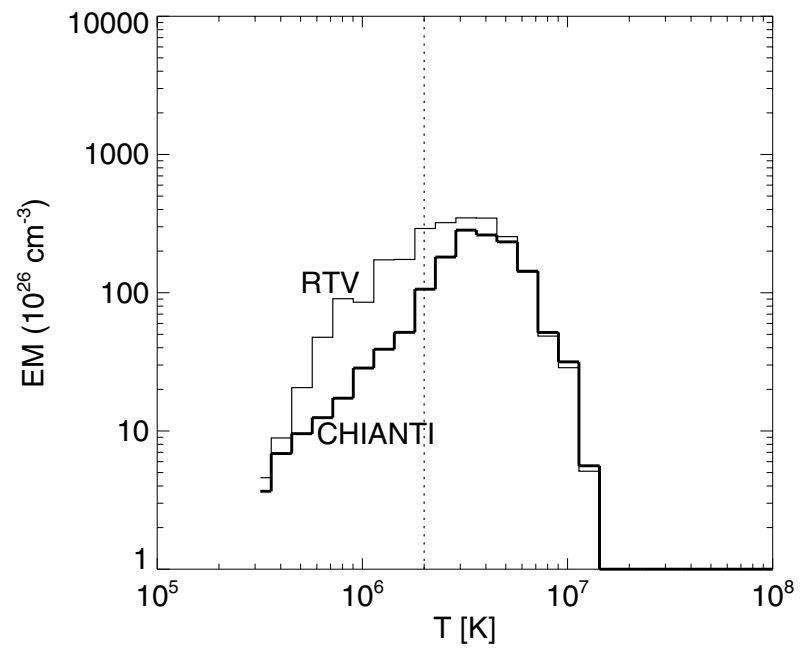

Fig. 5. $\operatorname{EM}(T)$ curves obtained by averaging the emission measure distribution versus temperature over the first $2000 \mathrm{~s}$ of strand evolution, obtained from the simulation using the RTV (thin solid line) and the CHIANTI (thick solid line) radiative losses. The temperature of $2 \mathrm{MK}$ is marked for reference (dotted vertical line).

coronal observations is the distribution of the emission measure as a function of temperature (hereafter $E M(T)$ )

$E M(T)=\int_{0}^{V(T)} n(T)^{2} \mathrm{~d} V$.

Typical coronal $\operatorname{EM}(T)$ distributions monotonically increase by a few orders of magnitude from $\sim 0.1$ to $\sim 1 \mathrm{MK}$, have a broad peak around 2-3 MK and then decrease again to higher temperature, more steeply than they rise (e.g., Peres et al. 2000; Testa et al. 2011; Warren et al. 2011). The $E M(T)$ curve of a coronal loop can be predicted from theoretical models of loop strands similar to the one we used here by assuming that the loop consists of many strands, each heated randomly in time. Thus, the overall $\operatorname{EM}(T)$ curve is determined by assuming that at any given time there will be loop strands at all stages of the strand evolution, and the total $E M(T)$ curve can be obtained by averaging the $\operatorname{EM}(T)$ curve of a single strand over the entire strand evolution, in this case $2000 \mathrm{~s}$.

In Fig. 5, we plot the space- and time-averaged $\operatorname{EM}(T)$ curves obtained from the simulations made using the RTV and the CHIANTI radiative losses, in the coronal part of the strand, i.e. excluding moss regions (Guarrasi et al. 2010; Warren et al. 2011). The figure shows that the high temperature portion of the $E M(T)$ curve does not change in the two calculations, confirming that using different radiative-loss curves does not change the results above 3-4 MK. In contrast, the low temperature part significantly differs, owing to the different slopes of the cooling at late times: the RTV simulation leads to a slightly higher peak and to a shallower distribution at the low temperature tail, that steepens below $\sim 0.5 \mathrm{MK}$. On the other hand, the faster cooling obtained with CHIANTI leads to a steeper curve below $2 \mathrm{MK}$, thus to a sharper peak at $3 \mathrm{MK}$. The absolute difference between the two curves reaches a factor of five around $1 \mathrm{MK}$; however, the $E M(T)$ slope itself has been indicated to be an important parameter in discriminating between low-frequency and high-frequency coronal heating mechanisms (e.g. Warren et al. 2011). In the temperature range $5.9 \leq \log T \leq 6.5$, the slope in the logarithmic scale is $\approx 1.1$ for the RTV curve, and $\approx 2.0$ for the CHIANTI curve. Although there is a general agreement, we 


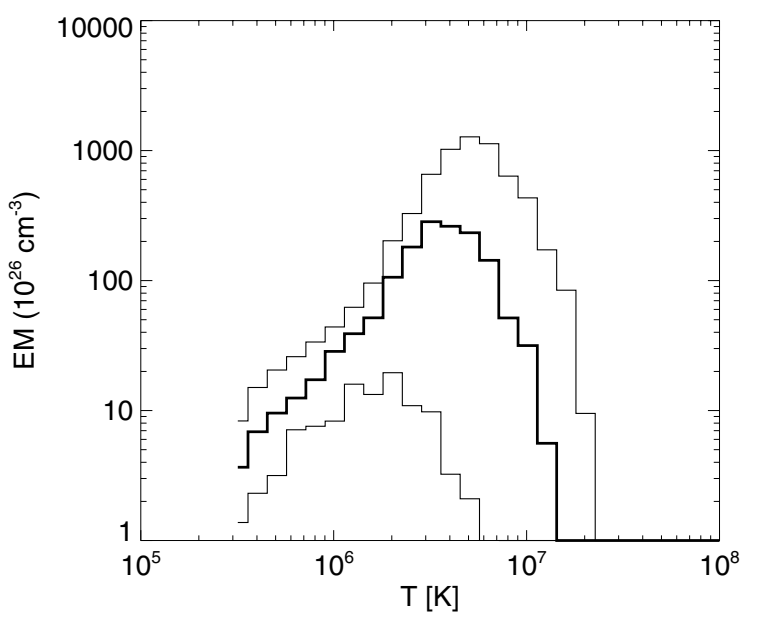

Fig. 6. $E M(T)$ curves obtained by averaging the emission measure distribution versus temperature over the first $2000 \mathrm{~s}$ of strand evolution, obtained from three simulations performed using CHIANTI radiative losses and three different heat pulse amplitudes, namely the same used in Fig. 5 (thick solid line), a lower one (lower thin solid line), and a larger one (upper thin solid line) (see also Fig. 4).

find that the slopes differ more than those found in Mulu-Moore et al. (2011). These values are shallower than those obtained by Warren et al. (2011), but one cannot exclude that they may be compatible when all possible uncertainties in the data analyses and issues regarding DEM reconstruction (Testa et al. 2011) are taken into account.

Moreover, the two different slopes on the cool side of the $E M(T)$ curves lead to different intensity ratios of the EUV lines being frequently observed by the available instrumentation (Warren et al. 2011). Figure 6 shows two more distributions obtained with CHIANTI radiative losses and different heat pulse intensities, one with a smaller $H_{1}$ value leading to a density steadily below the threshold value in Eq. (10), the other with a larger $H_{1}$ leading to a very high density (Fig. 4). The figure clearly shows that the trend on the cool side of the $\operatorname{EM}(T)$ becomes shallower and more similar to the RTV results at low density: this means that i) the different slope is linked to the presence of different cooling rates; and ii) it can in principle be used to efficiently discriminate between coronal heating rates and mechanisms.

The faster cooling with CHIANTI losses below $2 \mathrm{MK}$ has a second, important implication. The plasma in the strand spends a much longer time at temperatures well above 1-2 MK than below them. If a loop consists of a multitude of strands, each heated once randomly, most of the strands within a loop will have a high temperatures at any given time, while only few of them can be observed at temperatures at or below $\sim 1 \mathrm{MK}$. This might explain, at least in part, why active regions have been found to be mostly covered by hot $\sim 3 \mathrm{MK}$ loops with X-ray images and SDO/AIA $335 \AA$ channel images, and much less populated by warm $\sim 1 \mathrm{MK}$ loops, as in the EUV $171 \AA$ channel in TRACE or SDO/AIA images, i.e. few loops are seen cooling from hot to warm status, which is much fewer than expected. To illustrate this effect, Fig. 7 shows synthetic images of a multi-stranded loop in the two SDO channels obtained from the hydrodynamic simulations. We consider 200 straight strands grouped in bundles of ten to mimic a limited instrument resolution. The straight aspect may recall a loop projected on the solar disk. Each strand has the evolution described above, but the start time of the heat pulse is shuffled: the strands brighten
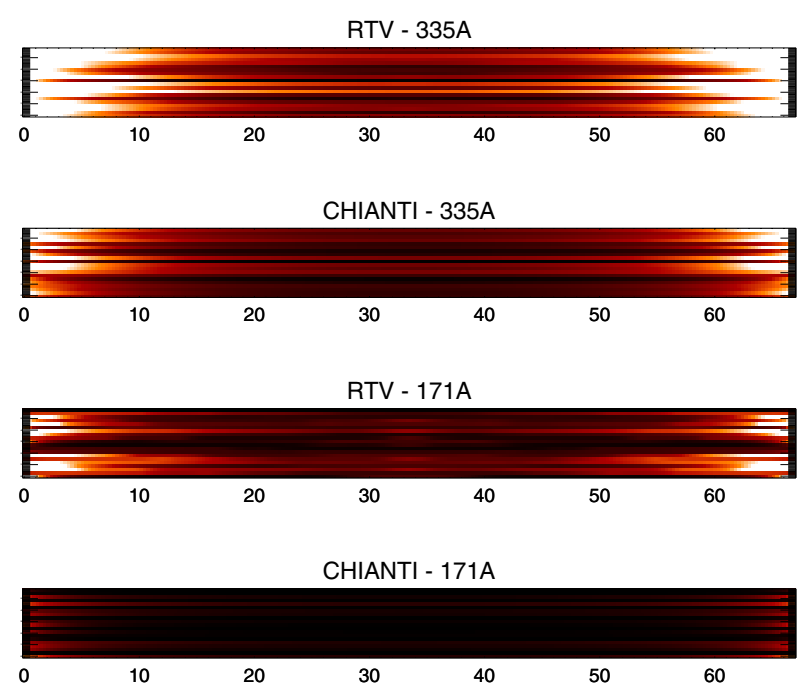

Fig. 7. Synthetic (straightened) loop images obtained from collecting groups of ten randomly heated strands, in SDO/AIA $335 \AA$ and $171 \AA$ channels, with models using RTV and CHIANTI radiative losses. The color scale is linear and exactly the same for a given channel (white is bright). The $X$-axis is measured in arcsec.

at random times. We take a snapshot in a regime situation, i.e. the emission spatially averaged over the stranded loop is equal to the average emission over the whole strand time-evolution. We show images obtained with both RTV and CHIANTI radiative losses. The color scale is linear and chosen to be exactly the same in each channel. In the $335 \AA$ channel, we see differences in the details but overall the loop appears to be bright. In the $171 \AA$ channel, there is a large qualitative change: the loop is much fainter, almost invisible, with the CHIANTI radiative losses. In a nutshell, the CHIANTI radiative losses significantly decrease the time that a given loop strand spends at a temperature between $1 \mathrm{MK}$ and $3 \mathrm{MK}$, so that on average it will be more difficult to observe loops in many narrow-band channels from AIA, TRACE, STEREO/EUVI and SOHO/EIT than expected when using RTV radiative losses. Since the onset of the accelerated (and then catastrophic) cooling depends on the plasma density, hence on the rate of impulsive heating, changes in the radiative losses can have significant effects on the heating rates determined from narrow-band images.

\section{Discussion and conclusions}

We have studied the effects of changes to the radiative-loss curve on the evolution of an impulsively heated loop strand. Radiative losses can change due to 1) improvements in atomic models with the inclusion of more accurate atomic data and transition rates, or lines previously unavailable; 2) upgrades in the plasma ion-abundance composition; 3) changes in the plasma elementabundance composition. We have carried out our tests using two radiative-loss curves, whose differences were due to all three of these causes. The most recent curve, from CHIANTI version 7, had much more efficient radiative losses at temperatures in the 0.5-3 MK range than the older one (RTV losses). We expect to find similar effects using other losses curves obtained from other spectral codes, with similar atomic models and element abundances (Sect. 1). More efficient radiative losses may have effects on modeling other thermally unstable optically thin plasmas, such as flares, supernova remnants, accretion columns from 
circumstellar disks, novae, and galactic cooling flows. Here we have focussed on their effects on the physics and structure of coronal loops, particularly the evolution of plasma confined inside a coronal magnetic flux tube that is subject to a short and strong heat pulse.

The effects are very small in the initial phases of the evolution. Most previous studies of the flaring plasma focused on the rise and initial decay phase of the flare, and much less on the very late phases, so their results are unaffected by changes in the radiative losses. In contrast, Reale et al. (2012) studied the EUV late phase of a flare using the older RTV radiative losses, and were able to reproduce the observed light curves with success. The agreement they found between model and observations suggests two possibilities. First, a small change in the model parameters may be enough to maintain the same degree of agreement if the CHIANTI radiative-loss function is used: for example, greater radiative losses may require a smaller amount of heating to lead to the final catastrophic cooling that explained the observations. Second, the element abundances in the flaring plasma may be lower than the coronal values assumed in the CHIANTI rates used in the present work so that the differences between the RTV and the CHIANTI radiative losses will be smaller.

Our results show that changes to the radiative-loss curves have important effects on both the late phases of a pulse-heated coronal loop strand, because they can change the cooling rate of the heated plasma, and the timing of the final catastrophic cooling. This can lead to immediate and interesting consequences for what we can expect from observations.

First, the change in the cooling rate occurs at relatively low temperature ( $\sim 2 \mathrm{MK})$, and leads to a considerable steepening of the cool side of the emission measure distribution. This change in the $E M(T)$ slope has important effects on our understanding of coronal plasma heating. Earlier studies of pulse-heated loop models, using RTV-like radiative losses provided relatively flat $E M(T)$ curves and these broad curves, which are not observed in active regions, have been invoked as an argument against the effectiveness of impulsive heating in non-flaring active regions (Warren et al. 2011). Thus, the change in slope caused by an improved radiative-loss function can lead to a significant change of perspective.

Second, the temperature of the cooling plasma decreases more rapidly below $2 \mathrm{MK}$, and this might explain why we see very few loops cooling from X-rays to EUV bands in active regions.

Third, we note that we do not expect faster cooling to occur in lower-temperature regime loops: if the loop is heated by less intense pulses or in a more gradual and longer-lasting way, the plasma may never start to cool rapidly. Whenever we have observed 1 MK loops in UV "warm" channels, this might provide an upper limit to the intensity and duration of the heat pulses that provide the energy.

Fourth, Reale et al. (2012) showed that a catastrophic cooling occurred at the end of the evolution of a post-flare loop, and explained the so-called EUV late phase observed by EVE during the decay of flares (Woods et al. 2011). Changes in radiative losses cause the predictions of the EUV late phase of flares to change considerably, and affect any estimate of flare impulsive heating rates made by combining cooling loop models with observed EUV light curves.

This work uses one the latest release of an up-to-date spectral model. However, chances are that spectral codes will continue to evolve and include increasingly more lines and more accurate data as they become available. For example, there is evidence that we may have yet to incorporate many important spectral lines (Testa et al. 2012) that can have significant effects on the radiative losses. As a consequence, we expect even more significant effects to be caused by further spectral model improvements, and the present work can even be considered conservative in this respect. In addition, element abundances are very important to radiative losses, so studies that provide tighter constraints on abundances are highly encouraged. In contrast, the effects of deviations from ionization equilibrium of the emitting species are negligible during the late phase of the flare, because the plasma is still of relatively high density, so that the ionization equilibrium times are quite short and the plasma is able to respond quickly and adapt to the rapid changes in the temperature (Reale et al. 2012).

In conclusion, we have reported on the effect of upgrading the spectral models of optically thin emitting plasmas used to model coronal plasma. In particular, we have found that, while the higher emissivity predicted by new spectral models has negligible effects on most of the plasma evolution predicted by loop models and in many plasma conditions, there are very important implications for the modeling of plasma confined inside coronal loop strands heated by strong and rapid energy pulses. Enhanced plasma emission in the 0.5-3 MK temperature range leads to faster cooling that could explain why some observations may appear inconsistent with pulse-heated loop models. We point out that the effects that we study here might also be important for modeling other thermally unstable optically thin plasmas, such as supernova remnants, accretion columns from circumstellar disks, and novae.

Acknowledgements. We thank Peter Cargill for useful suggestions and the anonymous referee for constructive comments. Fabio Reale acknowledges support from Italian Ministero dell'Università e Ricerca, from Agenzia Spaziale Italiana (ASI), ASI/INAF agreement I/023/09/0 and from the International Space Science Institute (ISSI) in the framework of an international working team on Coronal heating (ID 210/2011). The work of Enrico Landi is supported by NASA grants NNX10AQ58G and NNX11AC20G, and by NSF grant AGS-1154443.

\section{References}

Allen, C. W. 1973, Astrophysical quantities (London: The Athlone Press)

Antiochos, S. K. 1980, ApJ, 241, 385

Antiochos, S. K., \& Sturrock, P. A. 1982, ApJ, 254, 343

Antiochos, S., MacNeice, P., Spicer, D., \& Klimchuk, J. 1999, ApJ, 512, 985

Betta, R., Peres, G., Reale, F., \& Serio, S. 1997, A\&AS, 122, 585

Betta, R., Peres, G., Reale, F., \& Serio, S. 2001, A\&A, 380, 341

Bradshaw, S., \& Cargill, P. 2005, A\&A, 437, 311

Bradshaw, S., \& Cargill, P. 2006, A\&A, 458, 987

Bradshaw, S., \& Mason, H. 2003, A\&A, 407, 1127

Bradshaw, S. J., \& Cargill, P. J. 2010, ApJ, 717, 163

Cargill, P. 1993, Sol. Phys., 147, 263

Cargill, P. 1994, ApJ, 422, 381

Cargill, P. 1995, in Infrared tools for solar astrophysics: What's next?, ed. J. M. J. Penn, 17

Cargill, P., \& Klimchuk, J. 2004, ApJ, 605, 911

Dere, K. 2009, A\&A, 497, 287

Dere, K. P., Landi, E., Mason, H. E., Monsignori Fossi, B. C., \& Young, P. R. 1997, A\&AS, 125, 149

Doschek, G., Boris, J., Cheng, C., Mariska, J., \& Oran, E. 1982, ApJ, 258, 373

Feldman, U. 1992, Phys. Scr., 46, 202

Feldman, U., Mandelbaum, P., Seely, J. F., Doschek, G. A., \& Gursky, H. 1992, ApJS, 81, 387

Field, G. B. 1965, ApJ, 142, 531

Foster, A., Ji, L., Smith, R., \& Brickhouse, N. 2012, ApJ

Gan, W., Zhang, H., \& Fang, C. 1991, A\&A, 241, 618

Guarrasi, M., Reale, F., \& Peres, G. 2010, ApJ, 719, 576

Hansteen, V. 1993, ApJ, 402, 741

Kaastra, J. S., Mewe, R., Liedahl, D. A., et al. 1996, A\&A, 314, 547

Klimchuk, J. 2006, Sol. Phys., 234, 41

Klimchuk, J., Patsourakos, S., \& Cargill, P. 2008, ApJ, 682, 1351

Landi, E., \& Landini, M. 1999, A\&A, 347, 401 
Landi, E., Del Zanna, G., Young, P. R., Dere, K. P., \& Mason, H. E. 2012, ApJ, 744, 99

Landini, M., \& Monsignori Fossi, B. C. 1970, A\&A, 6, 468

MacNeice, P. 1986, Sol. Phys., 103, 47

McClymont, A. N., \& Canfield, R. C. 1983, ApJ, 265, 497

Müller, D., Hansteen, V., \& Peter, H. 2003, A\&A, 411, 605

Mulu-Moore, F. M., Winebarger, A. R., \& Warren, H. P. 2011, ApJ, 742, L6

Nagai, F. 1980, Sol. Phys., 68, 351

Nagai, F., \& Emslie, A. 1984, ApJ, 279, 896

Parker, E. 1988, ApJ, 330, 474

Parker, E. N. 1953, ApJ, 117, 43

Patsourakos, S., \& Klimchuk, J. 2005, ApJ, 628, 1023

Peres, G., Serio, S., Vaiana, G., \& Rosner, R. 1982, ApJ, 252, 791

Peres, G., Reale, F., Serio, S., \& Pallavicini, R. 1987, ApJ, 312, 895

Peres, G., Reale, F., \& Serio, S. 1993, in Physics of Solar and Stellar Coronae: G.S. Vaiana Memorial Symposuim, eds. J. Linsky, \& S. Serio (Dordrecht; Boston: Kluwer), Astrophys. Space Sci. Lib., 183, 151

Peres, G., Orlando, S., Reale, F., Rosner, R., \& Hudson, H. 2000, ApJ, 528, 537 Raymond, J. C., \& Smith, B. W. 1977, ApJS, 35, 419
Reale, F. 2007, A\&A, 471, 271

Reale, F. 2010, Liv. Rev. Sol. Phys., 7, 5

Reale, F., \& Orlando, S. 2008, ApJ, 684, 715

Reale, F., Peres, G., Serio, S., et al. 2000, ApJ, 535, 423

Reale, F., Guarrasi, M., Testa, P., et al. 2011, ApJ, 736, L16

Reale, F., Landi, E., \& Orlando, S. 2012, ApJ, 746, 18

Rosner, R., Tucker, W., \& Vaiana, G. 1978, ApJ, 220, 643

Soler, R., Ballester, J. L., \& Parenti, S. 2012, A\&A, 540, A7

Terzo, S., Reale, F., Miceli, M., et al. 2011, ApJ, 736, 111

Testa, P., Reale, F., Landi, E., DeLuca, E. E., \& Kashyap, V. 2011, ApJ, 728, 30

Testa, P., Drake, J. J., \& Landi, E. 2012, ApJ, 745, 111

Tucker, W. H., \& Gould, R. J. 1966, ApJ, 144, 244

Tucker, W. H., \& Koren, M. 1971, ApJ, 168, 283

Vernazza, J., Avrett, E., \& Loeser, R. 1981, ApJS, 45, 635

Viall, N. M., \& Klimchuk, J. A. 2011, ApJ, 738, 24

Warren, H., Winebarger, A., \& Hamilton, P. 2002, ApJ, 579, L41

Warren, H., Winebarger, A., \& Mariska, J. 2003, ApJ, 593, 1174

Warren, H. P., Brooks, D. H., \& Winebarger, A. R. 2011, ApJ, 734, 90

Woods, T. N., Hock, R., Eparvier, F., et al. 2011, ApJ, 739, 59 